\title{
The mQTL hotspot on linkage group 16 for phenolic compounds in apple fruits is probably the result of a leucoanthocyanidin reductase gene at that locus
}

\author{
Sabaz Ali Khan ${ }^{1,4}$, Jan G Schaart ${ }^{1}$, Jules Beekwilder², Andrew C Allan, Yury M Tikunov¹, Evert Jacobsen
} and Henk J Schouten ${ }^{1 *}$

\begin{abstract}
Background: Our previous study on ripe apples from a progeny of a cross between the apple cultivars 'Prima' and 'Fiesta' showed a hotspot of mQTLs for phenolic compounds at the top of LG16, both in peel and in flesh tissues. In order to find the underlying gene(s) of this $\mathrm{MQTL}$ hotspot, we investigated the expression profiles of structural and putative transcription factor genes of the phenylpropanoid and flavonoid pathways during different stages of fruit development in progeny genotypes.

Results: Only the structural gene leucoanthocyanidin reductase (MdLAR1) showed a significant correlation between transcript abundance and content of metabolites that mapped on the MQTL hotspot. This gene is located on LG16 in the $\mathrm{MQTL}$ hotspot. Progeny that had inherited one or two copies of the dominant MdLAR1 alleles (Mm, MM) showed a 4.4- and 11.8-fold higher expression level of MdLAR1 respectively, compared to the progeny that had inherited the recessive alleles $(\mathrm{mm})$. This higher expression was associated with a four-fold increase of procyanidin dimer II as one representative metabolite that mapped in the MQTL hotspot. Although expression level of several structural genes were correlated with expression of other structural genes and with some MYB and bHLH transcription factor genes, only expression of MdLAR1 was correlated with metabolites that mapped at the $\mathrm{mQTL}$ hotspot. MdLAR1 is the only candidate gene that can explain the mQTL for procyanidins and flavan-3-ols. However, mQTLs for other phenylpropanoids such as phenolic esters, dihydrochalcones and flavonols, that appear to map at the same locus, have so far not been considered to be dependent on LAR, as their biosynthesis does not involve LAR activity. An explanation for this phenomenon is discussed.
\end{abstract}

Conclusions: Transcript abundances and genomic positions indicate that the mQTL hotspot for phenolic compounds at the top of LG16 is controlled by the MdLAR1 gene. The dominant allele of the MdLAR1 gene, causing increased content of metabolites that are potentially health beneficial, could be used in marker assisted selection of current apple breeding programs and for cisgenesis.

Keywords: Phenylpropanoid pathway, Flavonoid pathway, Transcript abundance, Apple fruits, Phenolic compounds, Leucoanthocyanidin reductase gene

\footnotetext{
* Correspondence: henk.schouten@wur.nl

${ }^{1}$ Wageningen UR Plant Breeding, P.O. Box 16, Wageningen 6700 AA, The Netherlands

Full list of author information is available at the end of the article
} 


\section{Background}

Apple (Malus $\times$ domestica Borkh) is an important source of many secondary metabolites known as phenolic compounds [1,2]. These phenolic compounds have various functions in the plant such as protection against ultra violet light [3]. The phenolic compounds such as procyanidins are polymers of flavan-3-ols. In plants they often function to prevent herbivory. They provide an astringent taste to foodstuffs and, at longer chain length, form complexes with proteins. Procyanidins are increasingly recognized for their beneficial effects on human health [4].

One of the important benefits of these compounds to consumers is their potential role against various human diseases such as cancer, coronary heart diseases, cardiovascular diseases, and diabetes [5,6].

Phenolic compounds are synthesised through the phenylpropanoid and flavonoid pathways. For procyanidins, the biosynthetic pathway largely overlaps with that of anthocyanins. These complex biochemical pathways involve a series of enzymes. Many of these enzymes, as well as the encoding genes have been functionally characterized [7-10]. The first committed step to procyanidins has been postulated to be carried out by leucocyanidin reductase (LAR) [11].

In our previous study [1] we genetically mapped phenolic compounds that were detected in peel and in flesh of ripe apple fruits. We detected a hotspot of QTLs of metabolites (mQTLs) at the top of LG16. The metabolites that mapped at this locus were procyanidins (flavan-3-ols and their polymers), and other phenolic compounds such as phenolic esters and flavonol- and dihydrochalcone derivatives. All these compounds belong to the phenylpropanoids, and one could therefore speculate that the mQTL is controlled by a biosynthetic gene from the phenylpropanoid pathway, or by a transcription factor controlling this pathway.

The aim of the present study was to unravel which gene controlled the phenylpropanoid mQTL hotspot in apple. The approach used involved an expression analysis of structural and transcription factor genes of the phenylpropanoid and flavonoid pathway. By looking closer at the draft sequence of the whole genome of the apple cultivar 'Golden Delicious' [12], the structural gene leucoanthocyanidin reductase (MdLAR1) and seven transcription factor genes were detected in the genetic window of the mQTL hotspot. Therefore the transcript abundances of these genes were investigated. In addition, expression profiles of the structural genes of the phenylpropanoid and flavonoid pathways outside of the mQTL hotspot were studied. A strong positive correlation between the expression level of the MdLAR1 gene and the level of metabolites that mapped at LG16 was observed. This was not found for any of the other genes studied.
This indicates that the $M d L A R 1$ gene is the major candidate gene controlling the mQTL hotspot on LG16. Further evidence is provided by the fact that the MdLAR1 gene is the only structural gene of the phenylpropanoid and flavonoid pathways that resides in the mQTL hotspot.

\section{Methods}

In this study, fruits from the segregating F1 population derived from the cross between the cultivars 'Prima' and 'Fiesta' were used. This population was used in our previous study too, in which the mQTL hotspot and other mQTLs were detected [1].

\section{Selection of genotypes and harvesting of fruits for gene expression studies}

We selected genotypes based on the clear genetic segregation of metabolite 'procyanidin dimer II' (Additional file 1). This metabolite belongs to the phenylpropanoid pathway and its concentration showed a clear segregation (Figure 1). It was mapped at the mQTL hotspot on LG16 as described by Khan et al. [1]. This metabolite was used as representative metabolite for all other metabolites that mapped at the mQTL hotspot. The progeny genotypes from the cross 'Prima' $\times$ 'Fiesta' were divided into two groups based on 'procyanidin dimer II' clear segregation, i.e. one group (Group A) having low content and another group (Group B) having high content of 'procyanidin dimer II' (Additional file 2). The trees were at full bloom from 26-30 ${ }^{\text {th }}$ April, 2010. Fruits from trees in the trial orchard located in Randwijk, the Netherlands were harvested at three developmental stages (Figure 2), eight fruits per tree for each developmental stage, and subsequently peeled off and were stored as described in our

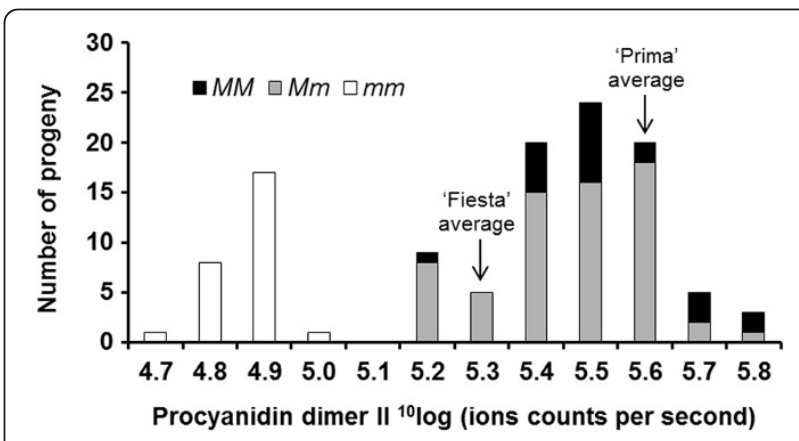

Figure 1 The procyanidin dimer II contents (ion counts per second) of the ripe fruits of the genotypes that were used for studying gene expression during fruit development [1]. This metabolite segregated clearly in the F1 progeny in both peel and flesh, and represents the metabolites that mapped in the mQTL hotspot on LG16. The genotype classes are $\mathrm{mm}$ (homozygous recessive), $M m$ (heterozygous dominant) and $M M$ (homozygous dominant). 


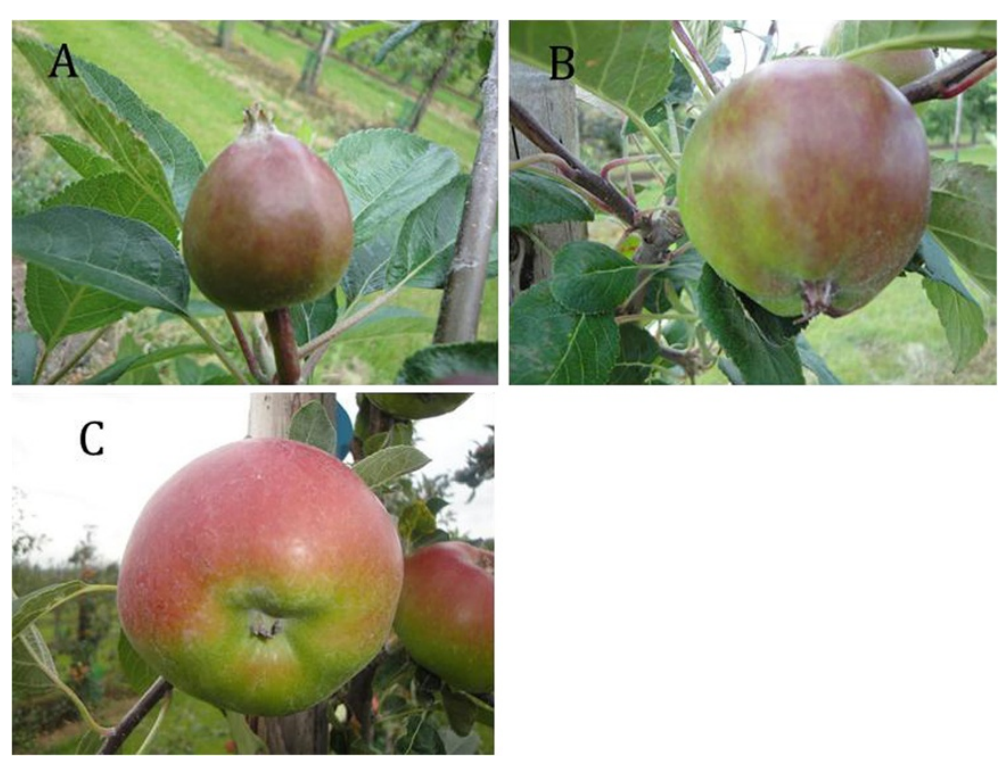

Figure 2 The three developmental stages of growth of apple fruit used for gene expression in this study. A= 34 Days After Full Bloom (DAFB), $\mathbf{B}=60$ DAFB and $\mathbf{C}=95$ DAFB.

previous article [1]. Ten genotypes were selected from 'Group A' and nine genotypes were selected from 'Group B' with two trees per genotype (two biological replicates; Additional file 1). As references, the parents of the segregating F1 population were included in the analysis with two trees for each parent. Both parents belong to the heterozygous class of genotypes. The genotype numbers and genotype classes used in this study are given in Additional file 1. The sizes (diameter) of individual fruits were measured at each developmental stage using an electronic digital caliper, model VWRI819-0012 of Control Company. The average size at 34 Days After Full Bloom (DAFB) was $24 \mathrm{~mm}$, at 60 DAFB $40 \mathrm{~mm}$, and at 95 DAFB $62 \mathrm{~mm}$ (Figure 2; Additional file 1).

There were three classes of genotypes based on cosegregating genetic markers: $M M$ was the homozygous dominant class. These progeny inherited from each parent one dominant allele for increased content of the procyanidin dimer II. $\mathrm{Mm}$ was the heterozygous class, which has one dominant allele from one parent and one recessive allele from the other parent. The heterozygous progeny had high content of the metabolite too. The third class is the homozygous recessive class $\mathrm{mm}$. This class received both recessive alleles from the two parents, and showed a low content of the metabolite.

\section{RNA isolation from apple fruits}

Total RNA was isolated from peel and flesh of apple fruits separately according to the CTAB method described by Asif et al. [13]. The RNA quantity was measured on NanoDrop ${ }^{\circledR}$ spectrophotometer model ND-1000 from isogen lifescience scientific company as explained by Khan et al. [14] and the RNA quality and quantity were measured by running $2 \mu \mathrm{l}$ of the RNA sample on a $1.5 \%$ agarose gel. First single-strand complementary DNA (cDNA) was synthesized using iScript ${ }^{\text {TM }}$ cDNA Synthesis Kit (Bio-Rad) according to the manufacturer's manual.

Selection of genes for qRT-PCR studies and primer design The MdLAR1 gene was detected in the middle of the genetic window for the mQTL hotspot at the top of LG16 [1]. Therefore this structural gene was included in the gene expression study. In addition, the other structural genes of the phenylpropanoid and flavonoid pathways were included (Table 1). Further more, all putative transcription factor genes that were located within the genetic window of the mQTL hotspot were included. Also we added putative transcription factor genes which neighboured this genetic window using the 'Golden Delicious' genome sequence [12], or which showed high homology to genes that are known to regulate the phenylpropanoid and flavonoid pathways in other plant species (Table 1). Primer pairs for structural genes of the phenylpropanoid and flavonoid pathways were kindly provided by Plant and Food Research, New Zealand. The primer pairs for the other candidate genes were designed with the online available program 'Primer3Plus' (http:// www.bioinformatics.nl/cgi-bin/primer3plus/primer3plus. cgi). The primer names and their forward and reverse 
Table 1 Genes that were included in the expression analysis

\begin{tabular}{|c|c|c|c|c|c|c|}
\hline Group & Gene name & Gene ID & Forward primer $\left(5^{\prime} \rightarrow 3^{\prime}\right)$ & Reverse primer $\left(5^{\prime} \rightarrow 3^{\prime}\right)$ & LG on "Golden Delicious" & Gene position on LG (kbp) \\
\hline \multirow{18}{*}{$\begin{array}{l}\text { Structural } \\
\text { genes }\end{array}$} & MdLAR1pair1 & MDP0000171928 & GTGGTTAACGGAGGCACAGT & CCGAGGAGAAAGGACTACCC & LG16 & 1536 \\
\hline & MdLAR1pair2 & AY830131 & GTGCTTCGATGGCTITCTTC & TAACAAGCTCACCCCCAAAC & LG16 & 1530 \\
\hline & MdLAR2 & AY830132 & ATGCCACAATCGTGTCAAAA & GGCTGGCTTCAGCTACAAAC & LG13 & 2860 \\
\hline & MdPAL & ES790093 & CGAGGAGTGTGACAAGGTGTTCCA & AGGAATGCAGCATGTAAACCGTGAC & LG4 & 8075 \\
\hline & $M d C 4 H$ & EB135197 & GGACGTTAAGTCCAGAACTTCGAGCT & ACTTCATCACAATGGTGGAATGCTTC & LG11 & 4614 \\
\hline & $\mathrm{Md} 4 \mathrm{CL}$ & EB122629 & CATAAACAGTGTCCCCAAGTCAGCAT & AGTGTTCCTACAAGCCTTCCCGATAA & LG11 & 5126 \\
\hline & MdCHS & CN944824 & GGAGACAACTGGAGAAGGACTGGAA & CGACATTGATACTGGTGTCTTCA & LG9 & 15948 \\
\hline & $\mathrm{MdCHI}$ & CN946541 & GGGATAACCTCGCGGCCAAA & GCATCCATGCCGGAAGCTACAA & LG1 & 16132 \\
\hline & MdF3H & CN491664 & TGGAAGCTTGTGAGGACTGGGGT & CTCCTCCGATGGCAAATCAAAGA & LG5 & 20985 \\
\hline & $M d D F R$ & AF117268 & GATAGGGTTTGAGTTCAAGTA & TCTCCTCAGCAGCCTCAGTTTCT & LG12 & 21890 \\
\hline & MdANS & AF117269 & GATGAAGGGAGGCTGGAGAAAG & GTGGAGGATGAAGGTGAGTGC & LG6 & 13776 \\
\hline & MdFLS & EB137300 & TCAGATGGAGATAATGAGCAATGGAAA & ATTAACGGGGTTCACAAGCTGTGG & LG8 & 15333 \\
\hline & MdANR & EB125405 & TCGCTGGCTTATGATCCTCCTGTT & CCGTTTGCCAAACTCAGCAAATTA & LG5 & 2243 \\
\hline & MdHCTchr9 & MDP0000851389 & CGATGCTGTTITCAGAACCA & GCAGCAGACGAGGATGATTA & LG9 & 24590 \\
\hline & MdC3Hchr8 & MDP0000466557 & CAAAGGAGGTGCTCAAGGAG & TGGACTCGACCATAGCAGTG & LG8 & 29024 \\
\hline & MdF3'Hchr6 & MDP0000539956 & ACTCTCTTCATGCGCTTGGT & TGCCTATCCTCACCCAAAAG & LG6 & 22805 \\
\hline & MdF3'Hchr14 & MDP0000370951 & ACCATTAAACCCCAACAACG & ATCACGGTTTGGAGCTCTTG & LG14 & 27562 \\
\hline & MdUFGT & AF117267 & AAGGTCTCTCCAATGTACGAAT & AGGAGTTTGTTGACTTTGGACT & LG1, LG7 & 29053,26292 \\
\hline \multirow{7}{*}{$\begin{array}{l}\text { TF genes at mQTL } \\
\text { hotspot }\end{array}$} & MdMYB1361 & MDP0000375685 & CTGGGGGTTCAAGTAGTCCA & CTCCGTGGTGGCTTGATAAT & LG16 & 1361 \\
\hline & Mdb-HLH1967 & MDP0000261293 & GATACGGCATCATTCCTGCT & GCCTGAGGATTTCCAACAAA & LG16 & 1967 \\
\hline & Mdb-HLH1881 & MDP0000154272 & CTCAACCGGGACTTATCCAA & GCTCATCCTCCCACACATTT & LG16 & 1881 \\
\hline & Mdb-HLH1543 & MDP0000319726 & GAGCTGAAACGCCAAACTTC & CGGTGATGAACAACACGTTC & LG16 & 1543 \\
\hline & MdAP21480 & MDP0000939633 & GCACCTTCAACGAAGAGGAC & GACTTGGAGTGGGAGCTCAG & LG16 & 1475 \\
\hline & MdG2L61440 & MDP0000202657 & AGACCGACTCCAACAATTCG & GGACTGGTGGTGAGACCTGT & LG13 & 2702 \\
\hline & MdbZIP1380 & MDP0000250967 & CTGTTTCTGGCAAAGGCTTC & CCATCAACATTGCAGTGGAC & LG16 & 1376 \\
\hline \multirow{8}{*}{$\begin{array}{l}\text { Transcription } \\
\text { factor genes } \\
\text { outside the } \\
\text { mQTL hotspot }\end{array}$} & MdCOL1220 & MDP0000185616 & TGATITTATGGGGTGCCAAT & TAATCACCGCCTCGTAATCC & LG16 & 1224 \\
\hline & Mdb-HLH1080 & MDP0000725991 & GGCCAATGACACCTCCTITA & TGAGCTGTGGAATGAGCAAC & LG16 & 1084 \\
\hline & MdMYB1070 & MDP0000659260 & ACTCCGCAAGAACAGCTCAT & GCTGTTCGACTCGATGTTCA & LG16 & 1058 \\
\hline & $\mathrm{MdC} 2 \mathrm{H} 21020$ & MDP0000183099 & ССТССТСАССТССТСТСТСС & CCCGGCTCTGTTGTAGTACC & LG16 & 1021 \\
\hline & $\mathrm{MdC} 2 \mathrm{H} 21000$ & MDP0000283750 & ATTCAGCAAGTTGGGTGTCC & TाTGCTTTGTGCAGTTGAGG & LG16 & 1003 \\
\hline & MdMyb5a.A & MDP0000791870 & GGGGAGGAGGAAATGAAGAG & CAGAGTCCCAGCCAAATGTT & LG3 & 967 \\
\hline & Mdb-HLH33 & MDP0000309179 & GGAGACATCAAAACCCGAAA & TGAAGGACATGCAAAGCAAG & LG15 & 37144 \\
\hline & MdTTG1 & MDP0000906307 & GACCCGGATACCCTTTCAAT & AAACTCGCTGGTCTTGCTGT & LG1 & 28763 \\
\hline
\end{tabular}


Table 1 Genes that were included in the expression analysis (Continued)

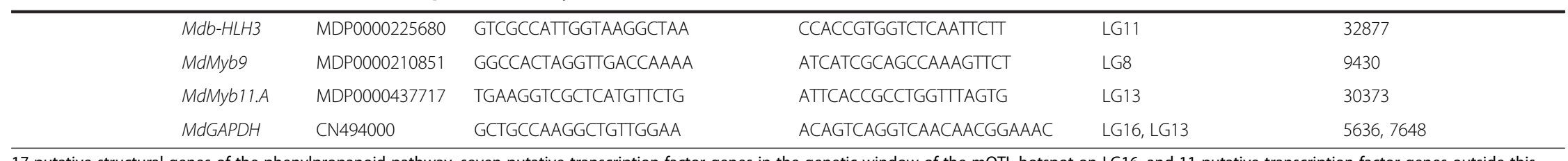

17 putative structural genes of the phenylpropanoid pathway, seven putative transcription factor genes in the genetic window of the mQTL hotspot on LG16, and 11 putative
mQTL hotspot were studied. MdGAPDH was used as a reference gene. The last four digits in some transcription factor genes represent their positions on the genome in kbp. 
sequences are given in Table 1. The primers were tested using q RT-PCR in the same way as explained by Khan et al. [14]. The qRT-PCR products were checked for quality by checking their clear single peak in the melting curve and a clear band of the expected amplicon size on $1.5 \%$ agarose gel.

In view of the importance of $M d L A R 1$, two primer pairs were designed for this gene in two different nonoverlapping regions. By means of these primer pairs, the two fragments were amplified and sequenced for each genotype class. This was done for verification of the gene specificity of the primers, since $M d L A R 1$ on LG16 and MdLAR2 on LG13 (Table 1 ) show $62 \%$ similarity at the nucleotide level. The sequences of $M d L A R 1$ and MdLAR2 in 'Prima' $\times$ 'Fiesta' showed good alignment with sequences from cv. 'Golden Delicious' on which the primers for qRT-PCR were designed.

\section{Performing q RT-PCR and data analysis}

Gene expression was measured using Fluidigm Dynamic Array integrated fluidic circuits for cDNA samples from peel and flesh for the genotypes and development stages mentioned in Additional file 1. Fluidigm used the BioMark ${ }^{\mathrm{Tm}}$ System and Evagreen DNA binding dye (http://www.fluidigm. com). Three 96×96 Dynamic Arrays of Integrated Fluidic Circuits, comprising 48 primer pairs in two replicates were used. The q RT-PCR set up for the reference gene and other control samples, and data analysis was performed as described by Khan et al. [14].

\section{Correlation network analysis}

The correlation coefficients were calculated between the contents of seven metabolites representative for different branches of the phenylpropanoid and flavonoid pathway, and for the expression of 18 structural genes and 18 transcription factors possibly involved in these pathways. Before calculation of the correlation coefficients, the data were ${ }^{10} \log$ transformed for normalisation purposes. Scatter plots were made between the different ${ }^{10} \log$ transformed variables, in order to make sure that outliers did not bias correlation values, and to check the distributions.

Visualization of the correlation network was performed by the Pajek software package (http://pajek.imfm.si/doku.php). Besides a biological quantitative pattern which is observed in a set of samples as the result of physiological processes, data may have a particular embedded 'experimental pattern' which is due to the experiment performance, such as extraction errors and measurement or calibration errors. So, different analytical methods run on the same set of samples may give different experimental patterns. Therefore, correlations between variables observed within particular experiments may be stronger than correlations between variables from different experiments. Here we have a correlation matrix of two different experiments and, therefore, three types of correlations (sub-matrices) are present: gene-to-gene correlations, metabolite-to-metabolite correlations and geneto-metabolite correlations. Lower correlation coefficients might be expected in the third sub-matrix due to interference of different experimental patterns. To compensate for this effect and to obtain a balanced correlation network we standardized correlation coefficients separately for each of the three sub-matrices. For these a maximum positive and negative correlation coefficients $r$ were found in each submatrix and then were set to 1.0 and -1.0 , respectively. Other correlation coefficients of each sub-matrix were expressed relative to their maximum ones. The standardized correlation coefficients are further denoted as $r_{\mathrm{s}}$.

\section{Results}

Association between expression of structural genes of the phenylpropanoid/flavonoid pathways and concentrations of metabolites that mapped at the MQTL hotspot

None of the 17 structural genes of the phenylpropanoid and flavonoid pathways which were evaluated for gene expression, showed a significant correlation with the content of procyanidin dimer II, except for the $M d L A R 1$ gene (Figure 3). This was observed both in peel and flesh tissues and at the three different fruit developmental stages (Additional file 3). For $M d L A R 1$ we evaluated the expression using two different primer pairs, annealing at different places in the $M d L A R 1$ gene. For both primer pairs, the measured expression showed a positive correlation with procyanidin and other phenolic metabolites that mapped in the mQTL hotspot (Figure 3). However, the metabolites quinic acid and coumaroyl hexoside appeared to have a negative correlation with the MdLAR1 expression (Figure 3).

The progeny that had inherited the recessive alleles for low procyanidin dimer II content $(\mathrm{mm})$, showed a low expression of MdLAR1 throughout fruit development, both in peel and flesh (Figure 2). However, the heterozygous group $(\mathrm{Mm})$ showed a higher expression, compared to the homozygous recessive $(\mathrm{mm})$ group, whereas the homozygous dominant progeny $(M M)$ with high content of procyanidin dimer II showed the highest expression of $M d L A R 1$ (Figure 2). The expression level of $M d L A R 1$ was highly significantly, positively correlated with procyanidin dimer II content, according to Student's t-test $(P<0.1 \%)$. On the average, the $M M$ genotypes had a four times higher content of this metabolite at the ripe stage compared to the $m m$ genotypes (Figure 1), both in peel and flesh.

No significant correlation was detected between transcript abundance of the other evaluated genes at the one hand with the concentration of procyanidin dimer II at the other hand (Figure 3).

The transcript abundance of $M d L A R 1$ was also significantly correlated with the other metabolites that mapped 


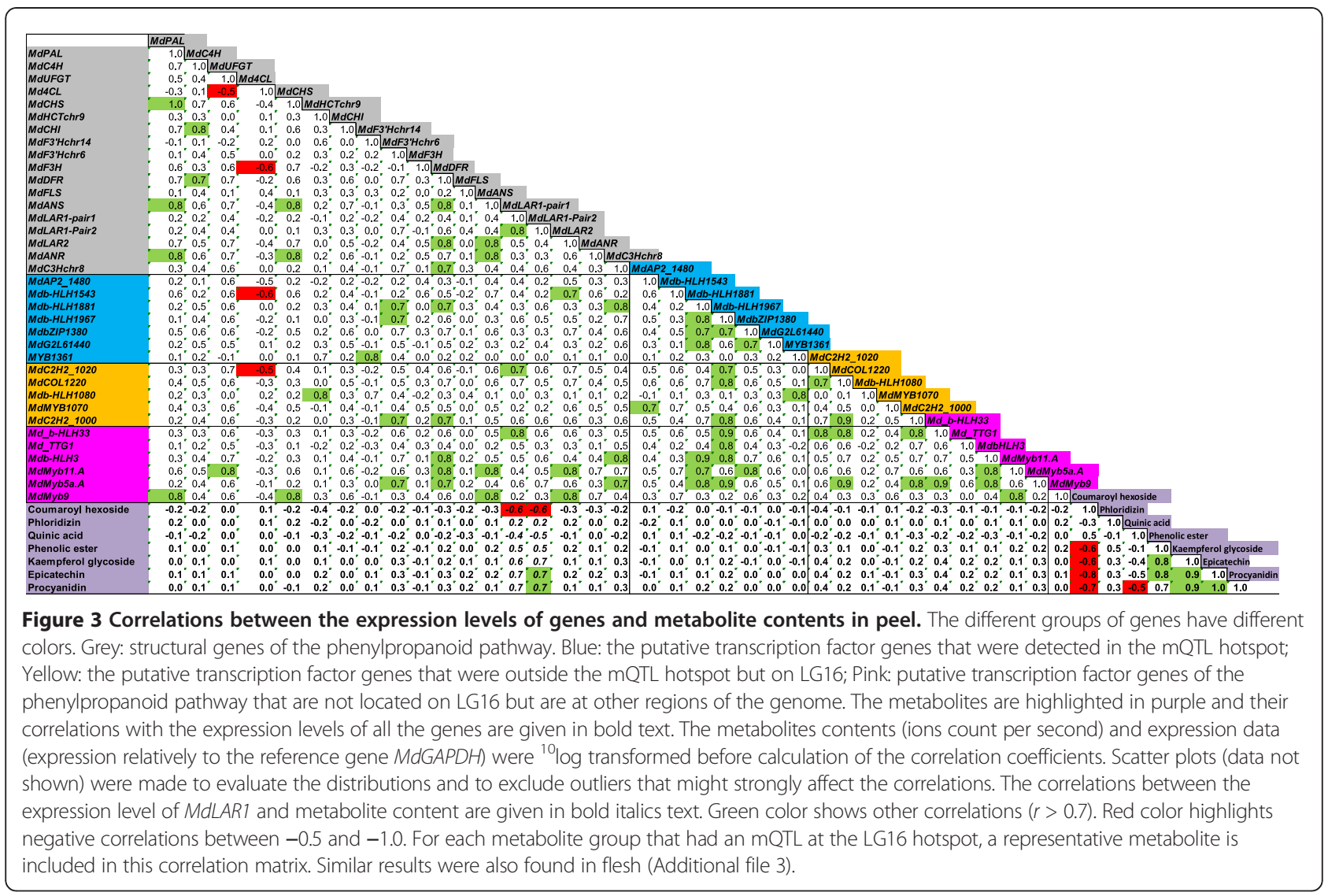

at the LG16 hotspot (Figure 3). However, the other studied genes did not show this high correlation with any of the metabolites at the mQTL hotspot. This suggests that the MdLAR1 gene is the gene controlling the mQTL of procyanidin dimer II, and of all other phenolic compounds that mapped at this hotspot on LG16.

\section{Association between expression of transcription factor genes and concentrations of metabolites that mapped at the mQTL hotspot}

The finding that compounds from different locations in the pathway mapped at the same mQTL hotspot [1] could suggest that a transcription factor was involved in the mQTL. At the mQTL locus, seven candidate transcription factor genes were identified (Table 1). However, there was no clear correlation for any of these candidate transcription factor genes with the procyanidin dimer II content in peel and flesh (Figure 3). This indicates that the evaluated transcription factor genes at the mQTL hotspot were not responsible for this hotspot.

In addition, 11 more candidate transcription factor genes were identified throughout the genome, or on homology to known transcription factors involved in the phenylpropanoid and flavonoid pathway (Table 1). No clear correlation was found between the expression of any of these putative transcription factor genes and the metabolites that mapped at the hotspot (Figure 3). This indicates that transcription factor genes outside the mQTL hotspot were not controlling this hotspot either.

\section{Associations between expression of structural genes and transcription factor genes}

The correlation matrix (Figure 3) shows that the expression levels of many genes were correlated to one another. As an example, the expression of the structural genes MdPAL, MdC4H, MdUFGT, MdCHS, MdCHI, $M d F 3 H, M d D F R, M d L A R 2$ and $M d A N S$ were positively correlated to one another. This cluster of structural genes showed also a positive correlation with the expression of the three transcription factor genes $b$-HLH1543, $M d M y b 11 . A$ and $M d M y b 9$. This suggests that these three transcription factor genes may regulate this cluster of structural genes, but did not control the mQTL hotspot on LG16.

We visualized the correlations of Figure 3 in a network (Figure 4). This network is divided into two clusters. The first cluster shows the correlations between the metabolites that mapped at the mQTL hotspot of LG16. The green lines in this cluster show positive correlations, and the red lines negative correlations. These colours resemble the mapping results, depicted in Figure 5. Striking in this cluster of metabolites is the presence of 


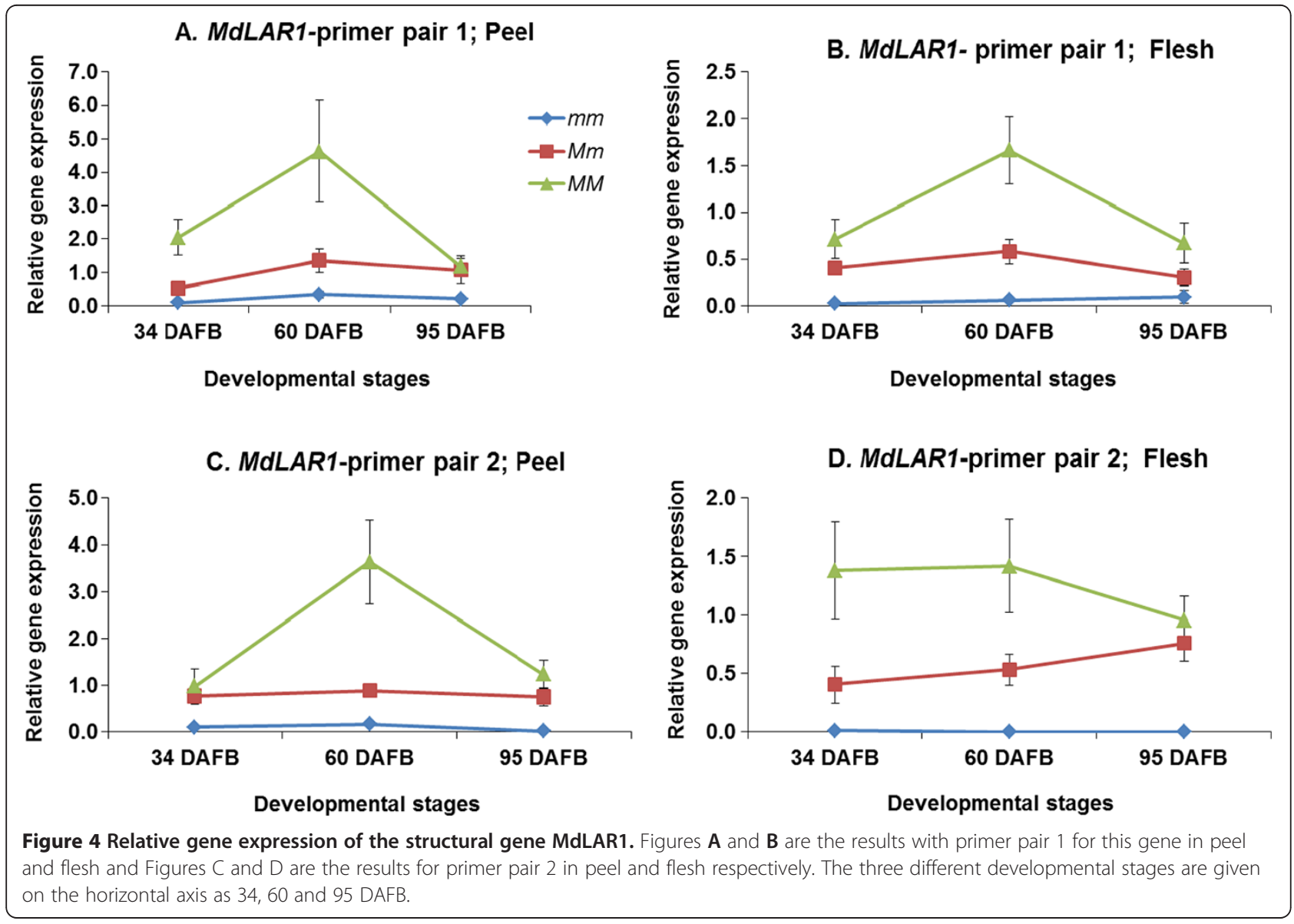

one gene only, i.e. MdLAR1. This gene is located in the centre of the MQTL hotspot of these metabolites.

In the second cluster of the network, many structural genes and transcription factors appear to be connected to one another (Figure 4). Several genes in the network are important nodes, and are connected to many other genes. This is especially the case for $M Y B$ transcription factors, such as $M d M Y B 9, M d M Y B 11 \_A$, and MdMYB5a_A, and for $b-H L H$ transcription factors, such as $b-H L H 1881$, $b$-HLH1967, and MdbHLH33 (Figure 4). Probably these transcription factors regulate many structural genes in the phenylpropanoid pathway. However, none of these transcription factor genes is directly connected to metabolites in the first cluster. In spite of the important regulatory roles of the mentioned MYB and $b-H L H$ transcription factor genes in the phenylpropanoid and flavonoid pathway, they were not responsible for the MQTL hotspot.

\section{Discussion}

\section{Aim of the study}

In our previous study [1] we mapped phenolic compounds in ripe fruits of a segregating F1 population derived from the cross between cultivars 'Prima' and 'Fiesta'. There appeared to be a strong hotspot of mQTLs at the top of
LG16. Annotation of the metabolites showed that the compounds that mapped on the LG16 hotspot belong to the phenylpropanoid and flavonoid pathways (Figure 5).

We wanted to discover which gene(s) controlled this mQTL hotspot. Therefore, in the present research, transcript abundances for the candidate genes in the mQTL region were measured in progeny genotypes that segregated for these mQTLs. In addition, structural genes of the phenylpropanoid and flavonoid pathways and putative transcription factor genes that are candidates for regulating these pathways and located elsewhere were evaluated as mentioned in the Methods section in detail.

MdLAR1 seems to be the only gene that can explain the mQTL hotspot on LG16

As shown in Figure 3, MdLAR1 was the only gene for which the expression was clearly correlated with the metabolite content, both in peel and flesh. None of the other genes showed a clear correlation with procyanidin dimer II content. Moreover, Figure 6 shows clearly that the expression of MdLAR1 was low for the genotypes that had inherited the recessive alleles $(\mathrm{mm})$, and had low content of the representative metabolite procyanidin 


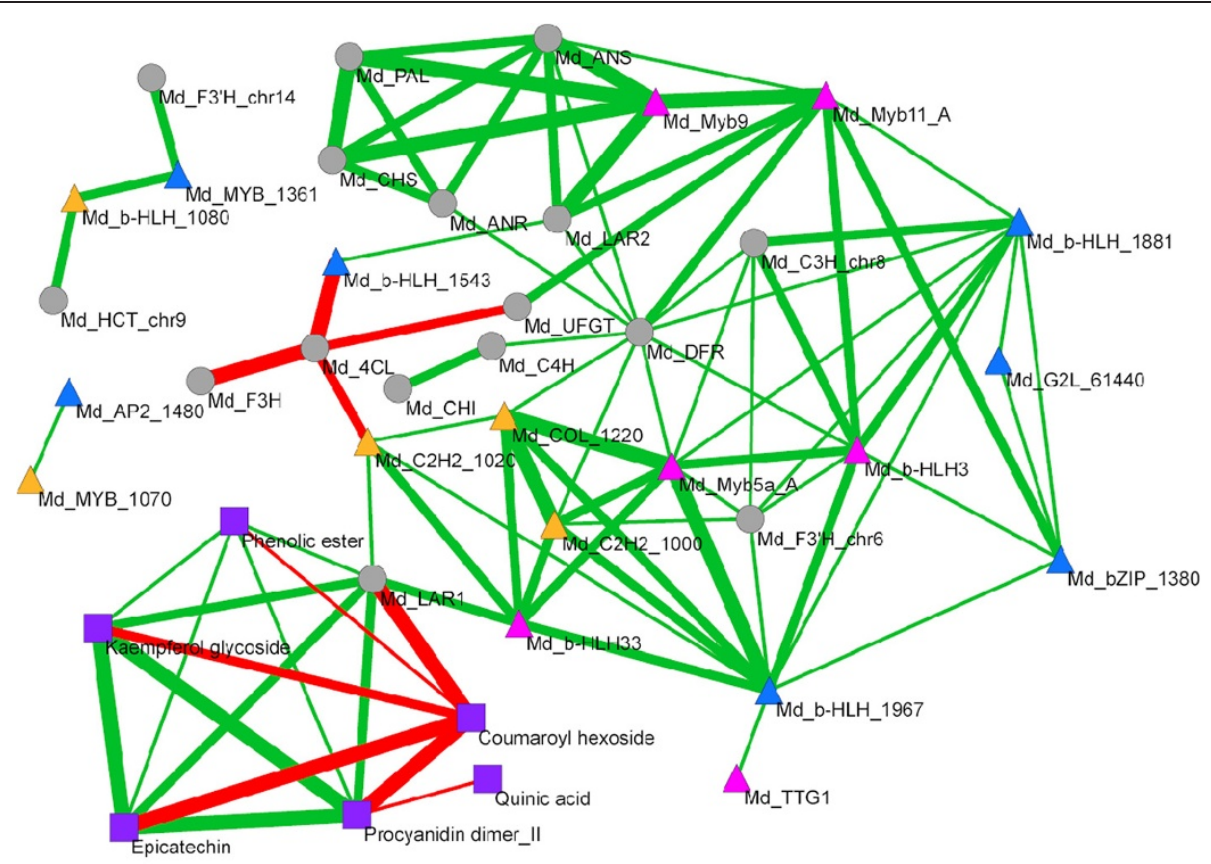

Red line - negative correlation

Box - metabolite Triangle - transcription factor Ellipse - structural gene

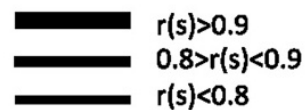

$r(s)<0.8$

Figure 5 A correlation network of 7 metabolites and 36 candidate genes of the phenylpropanoid and flavonoid pathways in apple peel. Different figures represent metabolites - purple box; structural genes - grey ellipse; transcription factors inside the mQTL hotspot on LG16 - blue triangle; transcription factors on LG16, but outside the mQTL hotspot - yellow triangle; transcription factors elsewhere in the apple genome - pink triangle. Red connection lines represent negative correlations and green lines - positive correlations and thickness of the lines correspond to the standardized correlation coefficient rs.

dimer II. The progeny that had inherited one or two dominant alleles $(M m, M M)$ had higher expression levels of $M d L A R 1$ and higher content of procyanidin dimer II (Figure 6). This pattern was observed both in peel and in flesh. This was not the case for any of the other genes studied, which suggests that $M d L A R 1$ was responsible for the hotspot of mQTLs on LG16. Furthermore, it indicates that $M d L A R 1$ exerted its influence by means of its expression level. Recent findings in grape also showed a genetic association between a $L A R$ gene and a procyanidin QTL [15].

The procyanidin content was higher in the flesh compared to the peel (Figure 1). However, the expression of MdLAR1 was lower in the flesh compared to the peel (Figure 6). A possible explanation is the fact that flavonols and anthocyanins are produced in the peel only. These may compete for the pool of available substrates, leading to relatively lower procyanidins level.

\section{How can MdLAR1 explain the observed mQTLs?}

The MdLAR1 gene clearly explains the mQTL for procyanidin content, as LAR from leguminosal species has been implicated in the synthesis of catechin, a building block for procyanidins [11]. Remarkably, we found several mQTLs in the same hotspot on LG16 for metabolites (kaempferol glycosides, phloridzin, phenolic esters) that are synthesized by different branches from the phenylpropanoid pathway [1] (Figure 5). Since $L A R$ is not known to be involved in the biosynthesis of these other metabolites, the observed differential $L A R$ expression does not provide a straightforward explanation for the presence of the mQTLs of these more upstream metabolites.

One could speculate about the effect that $L A R$ overexpression may have effect on the total flux through the phenylpropanoid pathway. We note that the positively associated mQTLs (procyanidins, dihydrochalcones, phenolic esters and kaempferol glycosides) all map downstream of coumaroyl-CoA ligase (4CL) in the pathway (Figure 5). A metabolite that maps upstream of 4CL is coumaroyl hexoside, for which the level was negatively correlated with e.g. procyanidins. This appears also from Figure 3.

In apple, no $4 C L$-like gene is located at the $\mathrm{mQTL}$ hotspot [12]. Moreover, the expression of the tested $4 C L$ gene did not correlate with the metabolites that mapped at the hotspot. One explanation may be that $M d L A R 1$ 


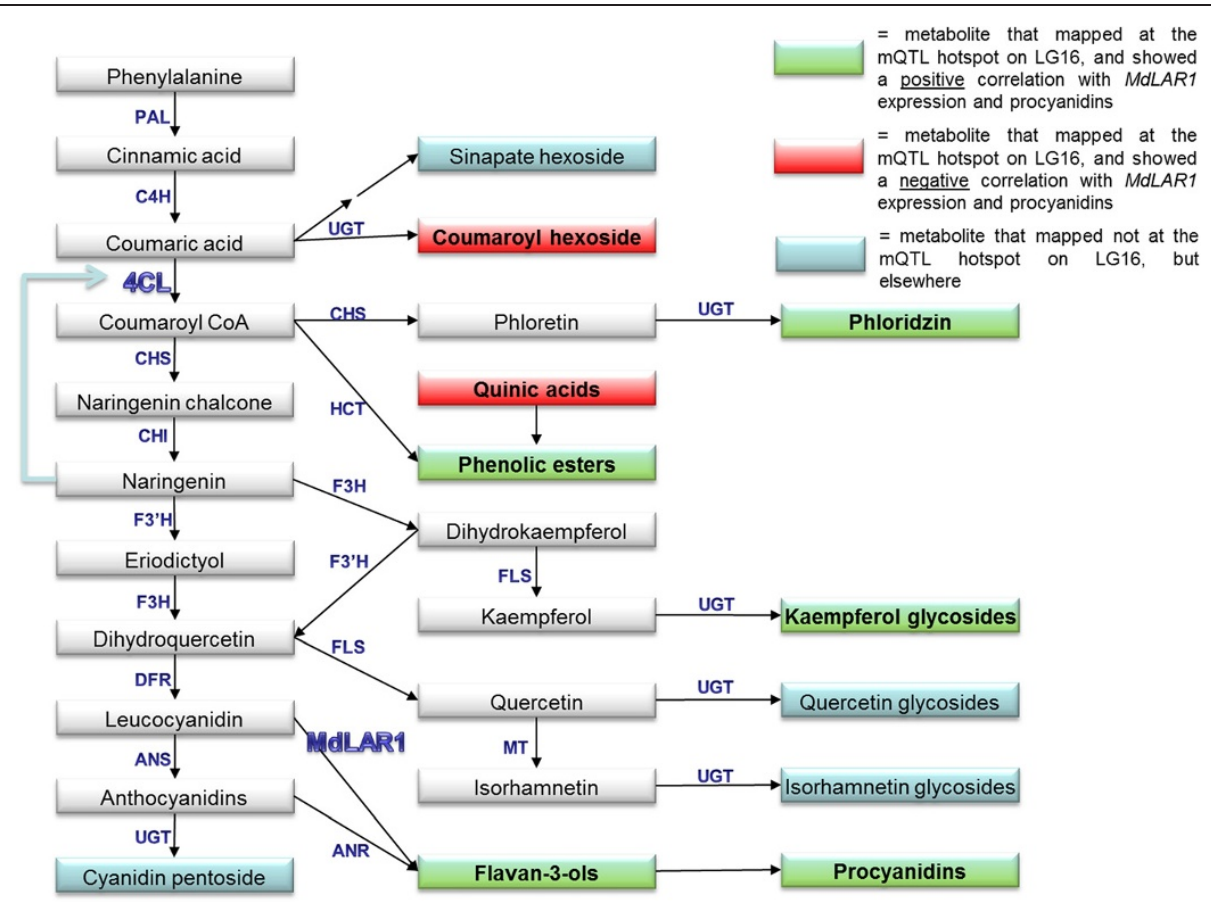

Figure 6 Simplified scheme of the mapping results of phenolic compounds in mature apple fruits according to Khan et al. [1].

overexpression relieves a feedback mechanism on the enzymatic activity of 4CL. 4CL is known to be feedback inhibited by metabolites from the phenylpropanoid pathway, such as naringenin [16]. Possi bly, the enhanced MdLAR1 activity will lead to depletion of pathway intermediates such as naringenin, which may thus activate 4CL activity and lead to a higher general flux, from coumaroyl glycoside towards the downstream metabolites. The support for such a mechanism needs extensive experimentation, which is outside the scope of this article.

An unlikely, but still possible alternative explanation for the mQTL hotspot could be that a transcription factor at the mQTL hotspot regulated the expression of $M d L A R 1$. As we did not see any differencial expression of the transcription factor genes at the mQTL hotspot, the different alleles of that transcription factor gene would not differ in expression levels, but theoretically could differ in effect of the protein. Further, that transcription factor might have influenced $4 C L$ paralogous that were not covered by the used primer pair. We do not regard this as a likely explanation, but it cannot be completely excluded.

\section{Transcript abundances of several structural genes and transcription factor genes were correlated}

MdANR also contributes to the synthesis of procyanidins (Figure 5). The expression level of this gene significantly correlated with expression of several structural genes such as PAL, CHS, DFR, and ANS (Figures 3 and 4).
Moreover, there was a clear correlation between the expression of these structural genes, and the expression of the transcription factor genes MYB9 and MYB11 (Figures 3 and 4). Possibly, these transcription factors regulated the mentioned structural genes. However, the transcript abundances of none of these structural or transcription factor genes did correlate significantly with the metabolite abundances that mapped at the mQTL hotspot on LG16 (Figure 3). This indicates that these structural genes were not the bottleneck for the pathway, whereas probably $M d L A R 1$ was the limiting factor in the progeny that had inherited both lowly expressed alleles of this gene $(\mathrm{mm})$. Presumably, the bottleneck was (partly) removed in case of presence of one or two higher expressed alleles of MdLAR1 (MM, Mm).

\section{Applications}

The dominant allele of the MdLAR1 gene, causing increased content of metabolites that are potentially health beneficial, could be used in marker assisted selection of current apple breeding programs. This selection could be made at seedling stage. This would reduce the production costs for the breeders by discarding the undesired seedlings at earlier stage of growth, whereas in classical breeding only after six years, when trees start to bear fruits, selection on fruit content is possible. Another possibility is to clone the dominant allele or alleles for engineering increased content of metabolite(s) into existing apple cultivars by different transformation technologies including cisgenesis $[17,18]$. 


\section{Conclusions}

Our results indicate that $M d L A R 1$ is the most likely candidate gene responsible for the mQTL hotspot for phenolic compounds on LG16 of apple, both in peel and flesh. Increased levels of metabolites downstream of MdLAR1, such as the flavan-3-ols epicatechin and procyanidin dimer II may be directly caused by increased transcript abundance of $M d L A R 1$, as this gene is known to participate in procyanidin biosynthesis.

\section{Additional files}

Additional file 1: Genotypes used for measuring relative expression of phenylpropanoid and flavonoid pathway genes and the candidate genes in the mQTL hotspot. Average sizes of eight fruits per tree genotype are also given at each stage.

Additional file 2: Genotypes used for measuring relative gene expression. 'Group A' had low and 'Group B' had high content of 'Procyanidin dimer II'. The content is given as ${ }^{10} \mathrm{log}$ transformed values of ions count per second.

Additional file 3: Corretions betwee the expression levels of genes and metabolite contents in flesh. The different groups of genes have different colours. Grey: structural genes of the phenylpropaniod pathway. Blue: the putative transcription factor genes that were detected in the mQTL hotspot; Yellow: the putative transcription factor genes that were outside the mQTL hotspot but on LG16; Pink: putative transcription factor genes of the phenylpropanoid pathway that are not located on LG16 but are at other regions of the genome. The metabolites are highlighted in purple and their correlations with the expression levels of all are given in bold text. The metabolites contents (ions count per second) and expression data (expression relatively to the reference gene MdGAPDH) were ${ }^{10} \log$ transformed before calculation of the correlation coefficients. Scatter plots (data not shown) were made to evaluate the distributions and to exclude outliers that might strongly affect the correlations. the correlations between the expression level of MdLAR1 and metabolite content are given in bold italics text. Light green colors shows other correlations $(r>0.7)$ between the expression levels of different genes and also between the expression of different genes and metabolites content. For each metabolite group that had a MQTL at the LG14 hotspot, a representative metabolite is included in this correlation matrix.

\section{Competing interests}

The authors declare that they have no competing interests.

\section{Authors' contributions}

SAK did the experimental work and have written down the manuscript. JGS and JB helped in the q RT-PCR studies. AA provided the primers for structural genes of the phenylpropanoid pathway. YT made the gene network. EJ provided useful ideas during experiment and the writing process. HJS initiated together with AGB this research, coordinated the project and helped in the writing of the article. All authors read and approved the final manuscript.

\section{Acknowledgements}

We are thankful to Higher Education Commission (HEC) of Pakistan for the fellowship funding and INOVA fruit B.V. for the financial support during this research.

\section{Author details}

'Wageningen UR Plant Breeding, P.O. Box 16, Wageningen 6700 AA, The Netherlands. ${ }^{2}$ Wageningen University and Research Centre, Plant Research International, Business Unit Bioscience, P.O. Box 16, Wageningen 6700 AA, The Netherlands. ${ }^{3}$ New Zealand Institute for Plant and Food Research Limited, Mt Albert Research Centre, Auckland 1025, New Zealand. ${ }^{4}$ Present address: Department of Environmental Sciences, COMSAT Institute of Information Technology, Abbottabad Postal code 22060, Pakistan.
Received: 16 September 2012 Accepted: 29 October 2012

Published: 2 November 2012

\section{References}

1. Khan SA, Chibon PY, de Vos RCH, Schipper BA, Walraven E, Beekwilder J, van Dijk T, Finkers R, Visser RG, van de Weg EW, et al: Genetic analysis of metabolites in apple fruits indicates an mQTL hotspot for phenolic compounds on linkage group 16. J Exp Bot 2012, 63(8):2895-2908.

2. Lu Y, Foo LY: Identification and quantification of major polyphenols in apple pomace. Food Chem 1997, 59(2):187-194.

3. Robberecht $R$, Caldwell MM: Leaf epidermal transmittance of ultraviolet radiation and its implications for plant sensitivity to ulraviolet-radiation induced injury. Oecologia 1978, 32(3):277-287.

4. Dixon RA, Xie DY, Sharma SB: Proanthocyanidins - a final frontier in flavonoid research? New Phytol 2005, 165(1):9-28.

5. Eberhardt MV, Lee CY, Liu RH: Antioxidant activity of fresh apples. Nature 2000, 405(6789):903-904

6. Mcghie TK, Hunt M, Barnett LE: Cultivar and growing region determine the antioxidant polyphenolic concentration and composition of apples grown in New Zealand. J Agric Food Chem 2005, 53(8):3065-3070.

7. Han Y, Korban SS: Genes encoding flavonoid $3^{\prime}$-hydroxylase in apple and their tagged molecular markers. Acta Horticulturae 2009, 839:409-414.

8. Jugde H, Nguy D, Moller I, Cooney JM, Atkinson RG: Isolation and characterization of a novel glycosyltransferase that converts phloretin to phlorizin, a potent antioxidant in apple. FEBS J 2008, 275(15):3804-3814.

9. Takos AM, Ubi BE, Robinson SP, Walker AR: Condensed tannin biosynthesis genes are regulated separately from other flavonoid biosynthesis genes in apple fruit skin. Plant Sci 2006, 170(3):487-499.

10. Kim SH, Lee JR, Hong ST, Yoo YK, An G, Kim SR: Molecular cloning and analysis of anthocyanin biosynthesis genes preferentially expressed in apple skin. Plant Sci 2003, 165(2):403-413.

11. Tanner GJ, Francki KT, Abrahams S, Watson JM, Larkin PJ, Ashton AR: Proanthocyanidin biosynthesis in plants - Purification of legume leucoanthocyanidin reductase and molecular cloning of its CDNA. J Biol Chem 2003, 278(34):31647-31656.

12. Velasco R, Zharkikh A, Affourtit J, Dhingra A, Cestaro A, Kalyanaraman A, Fontana P, Bhatnagar SK, Troggio M, Pruss D, et al: The genome of the domesticated apple (Malus x domestica Borkh). Nat Genet 2010, 42(10):833.

13. Asif $M H$, Dhawan $P$, Nath $P: A$ simple procedure for the isolation of high quality RNA from ripening banana fruit. Plant Molecular Biology Reporter 2000, 18(2):109-115.

14. Khan SA, Beekwilder J, Schaart JG, Mumm R, Soriano JM, Jacobsen E, Schouten HJ: Differences in acidity of apples are probably mainly caused by a malic acid transporter gene on LG16. Tree genetics and genomes 2012, doi:10.1007/s11295-012-0571-y.

15. Huang YF, Doligez A, Fournier-Level A, Le Cunff L, Bertrand Y, Canaguier A, Morel C, Miralles V, Veran F, Souquet JM, et al: Dissecting genetic architecture of grape proanthocyanidin composition through quantitative trait locus mapping. BMC Plant Biol 2012, 12:30.

16. Voo Kui S, Whetten RW, O'Malley DM, Sederoff RR: 4-Coumarate: Coenzyme A ligase from loblolly pine xylem. Isolation, characterization, and complementary DNA cloning. Plant Physiol 1995, 108(1):85-97.

17. Schouten HJ, Krens FA, Jacobsen E: Cisgenic plants are similar to traditionally bred plants: International regulations for genetically modified organisms should be altered to exempt cisgenesis. EMBO Rep 2006, 7(8):750-753.

18. Schouten HJ, Krens FA, Jacobsen E: Do cisgenic plants warrant less stringent oversight? Nat Biotechnol 2006, 24(7):753.

doi:10.1186/1756-0500-5-618

Cite this article as: Khan et al: The mQTL hotspot on linkage group 16 for phenolic compounds in apple fruits is probably the result of a leucoanthocyanidin reductase gene at that locus. BMC Research Notes 2012 5:618. 